\title{
Estudio piloto sobre la eficacia de una intervención para reducir la homofobia en hombres y mujeres adolescentes
}

\author{
Maria Astasio Centelles \\ al287472@uji.es \\ Naiara Martínez Gómez \\ al225840@uji.es \\ María Jesús Endrino Vilanova \\ maria.endrino@uji.es \\ Estefanía Ruíz-Palomino \\ eruiz@uji.es \\ Cristina Giménez García \\ gimenezc@uji.es
}

\section{Resumen}

La homofobia, a día de hoy, es todavía un problema prevalente en la mayoría de países del mundo, entre ellos España. Esto llega a tener graves implicaciones para la salud de aquellos que la sufren. Por ello, el presente estudio tiene como objetivo general validar la eficacia de un programa piloto para la reducción de la homofobia en adolescentes españoles. Con este fin, se realizó un taller, compuesto de tres sesiones, y se administró una escala de homofobia antes y después del mismo, con la finalidad de analizar si se habían producido cambios. Para ello, participaron 32 estudiantes de $2 .^{\circ}$ de Bachillerato de Ciencias de la Salud del IES Benigasló (La Vall d'Uixò, Castellón) con edades comprendidas entre 17 y 18 años; 11 eran chicos y 21 eran chicas. En general, los participantes presentan actitudes negativas hacia la homosexualidad en aspectos relacionados con las tres dimensiones que componen la escala (cognitiva, evitativa y agresiva), observándose un mayor grado de homofobia en los hombres, en comparación con las mujeres, en el caso de la dimensión cognitiva. Asimismo, al comparar las puntuaciones pretrest y postest, se observa que el programa piloto ha reducido los niveles de homofobia en los participantes, también en aspectos de las tres dimensiones, presentando una mayor eficacia en el caso de las chicas para la dimensión agresiva.

Palabras clave: homofobia, adolescentes, intervención, cognición, evitación, agresión. 


\begin{abstract}
Today, homophobia is still a prevalent problem in most of the countries of the world, including Spain. This has serious health implications for those who are suffering it. Therefore, the present study aims to validate the effectiveness of a pilot program for reducing homophobia in Spanish adolescents. To achieve this, a program composed of three sessions was conducted. In order to analyze if there have been any changes, a Homophobia Scale was administered before and after it. For this, 32 Senior High School students of Health Sciences belonging to Benigasló High School (La Vall d'Uixò, Castellón) participated. They were between 17 and 18 years old; 11 were boys and 21 were girls. In general, participants reveal negative attitudes towards homosexuality in aspects related to the three dimensions included in the scale (cognitive, avoidant and aggressive). In case of the cognitive dimension, men report higher degree of homophobia than women. Likewise, when comparing pre and post-test scores, the pilot program has reduced the levels of homophobia for all three dimensions. At the same time, it has also presented a great efficiency in girls within the aggressive dimension.
\end{abstract}

Keywords: homophobia, adolescents, intervention, cognition, avoidance, aggression.

\title{
Introducción
}

El concepto de homofobia hace referencia al miedo y la aversión hacia las personas homosexuales. En la actualidad, aunque es cierto que España cuenta con una legislación antidiscriminatoria hacia distintos colectivos, entre los cuales se ecuentra el LGTB (lesbianas, gays, transexuales y bisexuales) y que, según el Pew Research Center (2013), nuestro país es donde mayor aceptación hay de la homosexualidad $(88 \%)$, ello no significa que en nuestra sociedad no persista la homofobia y otras formas de discriminación hacia las personas LGTB.

Por lo tanto, a pesar de las mejorías encontradas en cuanto a la tolerancia hacia la homosexualidad a lo largo de estos años, es necesario ir más allá, especialmente en el contexto educativo, ya que, tal como muestra uno de los últimos informes (Benítez, Guilló y Vela 2015) un $32 \%$ de los alumnos pensaban que gays, lesbianas, bisexuales y transexuales eran abiertamente discriminados en las aulas. A este dato se suman otros como que un $7 \%$ del alumnado ha sido testigo de agresiones físicas por motivos homófobos y que este porcentaje aumenta a un $60 \%$ al hablar de agresiones verbales. Asimismo se señala que un $80 \%$ de los menores no heterosexuales deciden mantener su orientación sexual en secreto, posiblemente debido a esta atmósfera discriminativa que se da en el día a día en sus aulas.

Teniendo en cuenta este contexto, el objetivo del presente estudio es validar la eficacia de un programa piloto para disminuir la homfobia en adolescentes españoles, teniendo en cuenta posibles diferencies entre hombres y mujeres. 


\section{Método}

El presente estudio se basó en un diseño de investigación pre-post. En concreto el instrumento de evaluación utilizado para valorar el cambio actitudinal fue la escala de homofobia de Wright, Adams y Bernat (1999). Esta es una escala tipo Likert con 5 opciones de respuesta que van del «Totalmente en desacuerdo» al "Totalmente de acuerdo» y con 25 ítems que evalúan tres dimensiones distintas: cogniciones negativas, evitación y agresión hacia las personas homosexuales. El cálculo de la consistencia interna de esta escala con la muestra utilizada dio un alpha de Cronbach de 0,869 , con lo cual podemos afirmar que cuenta con una buena fiabilidad.

Por lo que hace referencia a la muestra, 32 adolescentes de 17 y 18 años, 34,37\% chicos y $65,63 \%$ chicas, participaron en el taller educativo destinado a reducir la homofobia. El programa consistía en tres sesiones, cuyas actividades se basaron en el aprendizaje participativo y en el trabajo grupal. En la primera sesión, «Reflexión sobre la homosexualidad», los estudiantes debatieron distintas oraciones con contenido homofóbico; en la segunda, «El mundo al revés», se visualizó y reflexionó sobre un cortometraje en el que se presentaba una sociedad donde se rechazaba la orientación heterosexual, la orientación mayoritaria era la homosexual y la minoritaría era la heterosexual; y, finalmente, en la tercera, "Cambiando el mundo», los alumnos debían pensar y elaborar estrategias para reducir la homofobia en la población actual. Las sesiones se llevaron a cabo en las propias aulas, en horario escolar, y cada una de ellas tuvo una duración de 55 minutos.

En cuanto a los análisis estadísticos, se empleó el SPSS (Statistical Package for Social Sciences, versión 22). En primer lugar, se llevaron a cabo análisis descriptivos de las tres dimensiones que componían la escala, para conocer las medias y las desviaciones típicas de cada una de ellas, así como la prueba $t$ de Student para evaluar las diferencias previas según el sexo. Tras ello, para examinar si existían diferencias significativas entre los niveles de homofobia antes y después de la intervención, se realizó la prueba t para muestras relacionadas para cada una de las dimensiones pre-post. También se calculó la d de Cohen para evaluar el tamaño del efecto de las diferencias significativas.

\section{Resultados}

A continuación se presentan los análisis diferenciales que se llevaron a cabo, por un lado, para determinar si existían diferencias significativas en los niveles de homofobia antes del taller en función del sexo y, por otro, para evaluar si se redujo la homofobia tras la intervención. 
Tabla 1.

Análisis diferencias de las dimensiones según el sexo.

\begin{tabular}{|lccccc|}
\hline & Sexo & Media & $\begin{array}{c}\text { Desviación } \\
\text { estándar }\end{array}$ & t & Sig. \\
\hline Dimensión cognitiva & Chico & 7,45 & 1,97 & $-2,18$ & 0,037 \\
& Chica & 9,71 & 3,12 & & \\
Dimensión evitativa & Chico & 17,10 & 4,46 & 0,14 & 0,887 \\
& Chica & 16,81 & 5,61 & & \\
Dimensión agresiva & Chico & 17,64 & 4,65 & 0,60 & 0,554 \\
& Chica & 16,57 & 4,65 & & \\
\hline
\end{tabular}

Tal como podemos ver en la tabla 1 , en la prueba $t$ de Student para muestras independientes, que se realizó comparando las medias entre los sexos para cada una de las dimensiones evaluadas con la escala de homofobia pretest, se encontraron diferencias significativas en cuanto al sexo únicamente en la dimensión cognitiva, siendo las mujeres las que presetan un menor nivel de homofobia en este nivel. El tamaño del efecto encontrado en este caso es alto $(d=-0,78$; IC $=-1,54 ;-0,03)$ (véase la tabla 1).

Tabla 2.

Análisis diferenciales entre pretest y postest para las dimensiones.

\begin{tabular}{|c|c|c|c|c|c|c|c|c|c|}
\hline & \multicolumn{2}{|c|}{ Pretest } & \multicolumn{2}{|c|}{ Postest } & \multirow[b]{2}{*}{$t$} & \multirow[b]{2}{*}{ Sig. } & \multirow[b]{2}{*}{ d } & \multirow{2}{*}{\multicolumn{2}{|c|}{ IC (95) }} \\
\hline & Media & $\begin{array}{c}\text { Desviación } \\
\text { estándar }\end{array}$ & Media & $\begin{array}{c}\text { Desviación } \\
\text { estándar }\end{array}$ & & & & & \\
\hline $\begin{array}{l}\text { Dimensión } \\
\text { cognitiva }\end{array}$ & 8,86 & 3,00 & 7,48 & 2,20 & 3,88 & 0,001 & $-0,448$ & 0,829 & $-0,066$ \\
\hline $\begin{array}{l}\text { Dimensión } \\
\text { evitativa }\end{array}$ & 16,89 & 5,41 & 13,89 & 2,95 & 3,76 & 0,001 & 0,539 & 0,923 & 0,149 \\
\hline $\begin{array}{l}\text { Dimensión } \\
\text { agresiva }\end{array}$ & 17,03 & 4,92 & 13,76 & 3,59 & 4,62 & 0,000 & $-0,647$ & $-1,05$ & $-0,247$ \\
\hline
\end{tabular}

Por lo que respecta la evaluación de cambios tras la realización del taller, en la prueba t para muestras relacionadas, que se realizó comparando las medias de cada par de dimensiones pre-post contenidas en la escala de homofobia, se obtuvieron diferencias significativas en todas ellas: dimensión cognitiva $(t=3,876, p<0,001)$, dimensión evitativa $(t=3,765, p<0,001)$ y dimensión agresiva $(t=4,623, p<0,000)$. Hay que destacar que la dimensión agresiva es la que muestra una mayor diferencia significativa antes y después del taller (véase la tabla 2). 
Estas diferencias significativas también se apoyan por el tamaño del efecto que, tal como podemos observar en la tabla 2, se acerca a la moderación para las tres dimensiones.

Finalmente, se realizó también el análisis de covarianza para determinar si el sexo influía en las puntuaciones obtenidas tras la aplicación del taller, teniendo en cuenta la puntuación previa de los participantes. El resultado que se obtuvo indicaba que el hecho de ser hombre o mujer solamente parecía influir en la dimensión agresiva $(F=4,81 ; p<0,044)$, en la que las chicas han mejorado más tras la intervención.

\section{Discusión y conclusiones}

En el presente estudio se puede observar cómo, tras la aplicación del programa piloto, se han reducido los niveles de homofobia entre los adolescentes participantes en relación con la dimensión cognitiva, evitativa y agresiva.

En primer lugar, hay que destacar que se encontraron actitudes negativas hacia la homosexualidad en la muestra general, en el pretest, en aspectos incluidos en cada una de las tres dimensiones que constituían la escala. Estos resultados serían congruentes con los datos recogidos por Generelo, López y Arroyo (2013), según los cuales un $76,38 \%$ de personas del colectivo LGTB eran discriminados por su orientación sexual en el contexto escolar, y por Benítez, Guilló y Vela (2015), según los cuales un $32 \%$ de alumnos LGTB eran abiertamente discriminados en el aula.

En segundo lugar, hay que destacar que los chicos presentan más actitudes negativas hacia la homosexualidad que las chicas exclusivamente en el caso de la dimensión cognitiva, de modo que en la dimensión evitativa y en la agresiva no existen diferencias entre ambos en los niveles de homofobia. Este resultado estaría relacionado con uno de los hallazgos encontrado por Johnson, Brems y Alford (1997), en el que vieron que los hombres expresaban creencias más débiles que las mujeres en cuanto a que la homosexualidad tiene una base genética, creyendo más firmemente que era un estilo de vida que puede ser alterado a través de terapia. Es decir, los hombres también presentarían mayor nivel de homofobia en la dimensión cognitiva que las mujeres, según este estudio.

En cuanto a la reducción de los niveles de homofobia en la muestra general tras la intervención, como ya se ha explicado, se puede afirmar que el programa ha sido eficaz en este punto, pues ha dado lugar a una disminución en aspectos tanto de la dimensión cognitiva como de la dimensión evitativa y agresiva. Resulta relevante destacar que el taller comparte características que han mostrado ser eficaces como son las dinámicas participativas o el énfasis en el componente actitudinal (Agustín 2009; Platero y Gómez 2008; Serrano 2009) .

En último lugar, hay que señalar que la intervención fue igual de eficaz para chicos que para chicas en las dimensiones cognitiva y evitativa, pero en la dimensión agresiva mostraría una mayor eficacia en el caso de las mujeres. Esto podría explicarse por el hecho de que los roles de género tradicionales suelen facilitar la expresión de la agresividad entre los hombres con mayor facilidad, lo cual podría estar influyendo y, por tanto, requerir un componente específico de trabajo (Abad y Flores 2010).

La valoración de estos resultados cabe tenerla en cuenta a partir de una serie de limitaciones. Entre ellas podemos nombrar la reducida población que compone la 
muestra en la que lo hemos aplicado, así como la necesidad de realizar un mayor número de seguimientos para comprobar si los cambios producidos gracias al taller se mantienen con el tiempo. De igual forma, resultaría necesario contar con grupos de control para poder asegurar la rigurosidad del proceso. Asimismo, resulta relevante nombrar el hecho de que las evaluaciones se llevaron a cabo de manera autoadministrada, con lo que no se sabe ciertamente si los evaluados son del todo sinceros en sus respuestas. En cualquier caso, este es un primer paso para implementar medidas eficaces para la prevención y la erradicación de la homofobia.

Finalmente, resulta de interés comentar que futuras líneas de investigación podrían ir dirigidas, precisamente con la finalidad de superar estas limitaciones, a impartir el taller a un mayor número de adolescentes, incrementando de este modo la muestra obtenida, y a realizar seguimientos tras la finalización del mismo, por ejemplo cada tres meses, para evaluar el mantenimiento de los cambios producidos gracias al programa. Del mismo modo, se podría tratar de incrementar el número de sesiones del taller, para analizar si el grado de reducción de la homofobia es mayor que el observado en el presente estudio si se aumenta su duración. De esta forma, podrían consolidarse los hallazgos encontrados en este estudio, que aportan una alternativa para reducir los niveles de discriminación en las aulas y así mejorar el bienestar global de las personas.

\section{Referencias bibliográficas}

Abad, Luisas y Juan Antonio Flores. 2010. Emociones y sentimientos: La construcción social del amor. Cuenca: Universidad de Castilla-La-Mancha.

Agustín, Santiago. 2009. Diversidad sexual en las aulas. Evitar el bullying o acoso homofóbico. Recuperado de: http://ceip.edu.uy/IFS/documentos/2015/sexual/materiales/Aula_PLURAL.pdf [Consultado el 3 de marzo de 2017].

Benítez, Eduardo, Sara Guilló y José A. Vela. 2015. LGBT-Fobia en las aulas 2015. Recuperado de: https://cogameduca.files.wordpress.com/2016/03/1-lgbt-fobiaen-las-aulas-2015-informe-completo-web.pdf [Consultado el 25 de enero de 2017].

Generelo, Jesús, Agustín López y Antonio Arroyo. 2013. Estudio 2013 sobre discriminación por orientación sexual y/o identidad de género de género en España. Recuperado de: http://www.felgtb.org/temas/laboral/documentacion/estudios/i/3773/632/estudio2013-sobre-discriminacion-por-orientacion-sexual-y-o-identidad-de-genero-enespana [Consultado el 28 de enero de 2017].

Johnson, Mark E., Christiane Brems y Pat Alford. 1997. «Personality Correlates of Homophobia». Journal of Homosexuality 34(1): 57-69.

Pew Research Center. 2013. The Global Divide on Homosexuality: Greater Acceptance in More Secular and Affluent Countries. Recuperado de: http://www.pewglobal.org/2013/06/04/the-global-divide-on-homosexuality/ [Consultado el 24 de enero de 2017].

Platero, Raquel y Emilio Gómez. 2008. Herramientas para combatir el bullying homofóbico. Madrid: Talasa.

Serrano, Gonzalo F. 2009. Guía contra la homofobia para centros educativos. Bilbao: A Fortiori.

Wright, Lester W., Henry E. Adams y Jeffery Bernat. 1999. «Development and Validation of the Homophobia Scale». Development and Validation of the Homophobia Scale 21(4): 337-347. 